\title{
Cooperative Sensing among Cognitive Radios
}

\author{
Shridhar Mubaraq Mishra, Anant Sahai and Robert W. Brodersen \\ School of Electrical Engineering and Computer Science \\ University of California, Berkeley, California 94704 \\ Email: $\{$ smm, sahai, rb\}@eecs.berkeley.edu
}

\begin{abstract}
Cognitive Radios have been advanced as a technology for the opportunistic use of under-utilized spectrum since they are able to sense the spectrum and use frequency bands if no Primary user is detected. However, the required sensitivity is very demanding since any individual Radio might face a deep fade. We propose light-weight cooperation in sensing based on hard decisions to mitigate the sensitivity requirements on individual radios.

We show that the "link budget" that system designers have to reserve for fading is a significant function of the required probability of detection. Even a few cooperating users $(\sim 10$ 20) facing independent fades are enough to achieve practical threshold levels by drastically reducing the individual detection requirements. Hard decisions perform almost as well as soft decisions in achieving these gains. Shadowing correlation limits these gains and hence a few independent users perform better than many correlated users.

Unfortunately, cooperative gain is very sensitive to adversarial/failing Cognitive Radios. Radios that fail in a known way (always report the presence/absence of a Primary user) can be compensated for by censoring them. On the other hand, radios that fail in unknown ways or may be malicious, introduce a bound on achievable sensitivity reductions. As a rule of thumb, if we believe that $\frac{1}{N}$ users can fail in an unknown way, then the cooperation gains are limited to what is possible with $N$ trusted users.
\end{abstract}

\section{INTRODUCTION}

Over the past years, traditional approaches to spectrum management have been challenged by new insights into the actual use of spectrum. In most countries, all frequencies have been completely allocated to specific uses. For example, the Federal Communication Commission's (FCC) frequency allocation chart (see Figure 1) indicates multiple allocations over essentially all of the frequency bands. Thus, within the current regulatory framework, spectrum appears to be a scarce resource. On the other hand, actual measurements indicate low utilization, (see spectrogram in Figure 1) especially in the $3-6 \mathrm{GHz}$ bands [1]. This view is also supported by studies conducted by the FCC's Spectrum Policy Task Force which have reported vast temporal and geographic variations in the usage of allocated spectrum [2] [3]. These measurements seriously question the efficiency of the current regulatory regime.

As the measurements clearly show, many who have been allocated frequency bands by the regulatory agency (Primary users) are not using them all of the time, at all places. At the same time, others may like to use spectrum locally, but do not have a right to use the corresponding frequencies. Therefore,
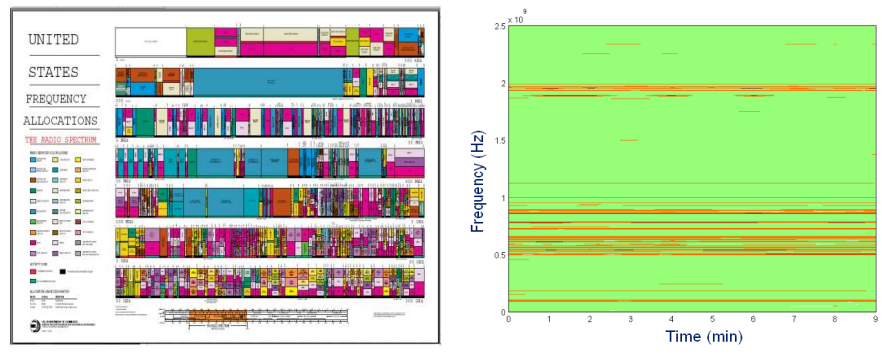

Fig. 1. FCC spectrum allocation and the measured usage at the Berkeley Wireless Research Center. The measurements were performed at $0-2 \mathrm{GHz}$ frequencies over a period of $10 \mathrm{mins}$.

one way of increasing spectrum efficiency is to enable these other secondary users to get access to frequency bands already allocated to Primary users while these are not using it. One of the ways of achieving this sharing ${ }^{1}$. is called Opportunistic Spectrum Sharing. Under such a regime, secondary users are allowed to operate in frequency bands without the consent of the Primary users of these bands, as long as they do not interfere with the Primary user. The FCC has already legalized this type of sharing in the $5 \mathrm{GHz}$ band and is considering whether to allow it in the TV broadcast bands [4].

\section{A. Cognitive Radios}

Cognitive Radios have been proposed as a technology to implement Opportunistic sharing since they are able to sense the spectrum and adapt their usage accordingly. Cognitive Radios must be able to demonstrate usage with no or minimal interference to the Primary user. This task is rendered difficult due to challenges in sensing the spectrum in a reliable manner. If a Cognitive Radio does not see energy in a particular band can it assume that the Primary user is not present? The answer depends on what level of energy it can reliably "see."

After all, a secondary user may suffer unlucky multipath and/or severe shadowing with respect to the primary transmitter. At the same time, it's own transmissions may interfere with a primary receiver should it decide to transmit. ${ }^{2}$ To account for possible losses from multipath, shadowing and building

\footnotetext{
${ }^{1}$ The primary other paradigm for spectrum sharing involves negotiated sublicensing of bands from primary users through either long term contracts, or the use of secondary "spot markets" in spectrum. We do not discuss that approach here

${ }^{2}$ This is related to the well known "hidden terminal problem" in wireless networking.
} 
penetration, the secondary user must be significantly more sensitive in detecting than the Primary receiver [5]. To get a better understanding of the problem, consider this: a typical Digital TV receiver operating in a $6 \mathrm{MHz}$ band must be able to decode a signal level of at least $-83 \mathrm{dBm}$ without significant errors [6]. The typical thermal noise in such bands is $-106 \mathrm{dBm}$. Hence a Cognitive Radio which is $30 \mathrm{~dB}$ better has to detect a signal level of $-113 \mathrm{dBm}$, which is below the noise floor. ${ }^{3}$

\section{B. The Motivation for Cooperative Sensing}

The two major sources of degraded signals are multipath and shadowing. For a given frequency, multipath varies significantly with a displacement of $\frac{\lambda}{4}$ as discussed in [7] (where $\lambda$ is the wavelength). Thus at $800 \mathrm{MHz}$, severe multipath can be avoided by displacing the antenna by $10 \mathrm{~cm}$ in a particular direction. In the absence of multiple antennas, multiple radios can act as a proxy for displacement or movement. The presence of multiple radios helps to reduce the effects of severe multipath at a single radio since they provide multiple independent realizations of related random variables. With multiple realizations, the probability that all users see deep fades is extremely low. In essence we wish to make Cognitive Radios' spectrum sensing robust to severe or poorly modelled fading environments. Cooperation allows us to achieve this robustness without drastic requirements on individual radios.

\section{Objectives and key insights}

Use of cooperation in wireless has been studied extensively especially in respect to achieving diversity gains and lowering outage probabilities via cooperation of mobile users [8]. In the Cognitive Radio context, we would like to exploit this cooperative effect in a different way. Rather than improving confidence by increasing cooperation, we want to maintain confidence while reducing competence! Hence our chosen metric is the reduction in sensitivity requirements once cooperation is employed (See Figure 2). Sensitivity of a radio is inherently limited by cost and delay requirements. Thus the device designer can figure out the implications of cooperation on the device specification through the well understood metric of detection sensitivity, thereby isolating the issue from unrelated concerns like the access regime, etc.

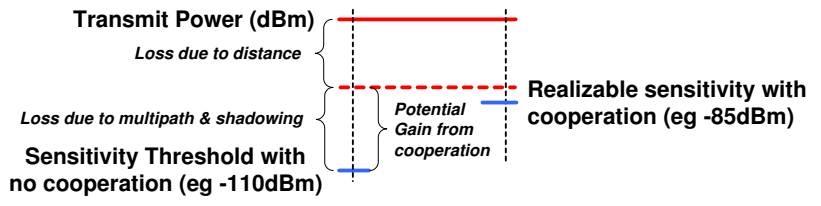

Fig. 2. Cooperation allows us to mitigate the effects of multipath and shadowing and hence the detection threshold can be set closer to the value of nominal path loss.

${ }^{3}$ Of course, being below the noise floor does not automatically make the detection problem impossible. The correlation structure of the primary transmissions, in particular the presence of known pilot tones, can make it possible to detect. However, it is always harder to detect weaker signals as compared to stronger ones.
In this paper, we show the following results:

- Cooperation allows independently faded radios to collectively achieve robustness to severe fades while keeping individual sensitivity levels close to the nominal path loss. Furthermore, a small number of radios $(\sim 10-20)$ are enough to achieve practical sensitivity levels.

- Practical "link budgets" for dealing with fading depend strongly on the target probability of detection which in turn depends on the tolerable probability for harmful interference at the Primary receiver and the number of non-cooperating Cognitive networks.

- Communicating tentative hard decisions can achieve cooperative gains nearly identical to sharing soft decisions.

- In a correlated fading environment, we cannot necessarily operate robustly with the sensitivity levels predicted by the analysis of independent users. In this case, polling a few independent users is better than polling many correlated users.

- Radios that fail in unknown ways or may be malicious, introduce a bound on achievable sensitivity reductions. As a rule of thumb, if we believe that a fraction $\frac{1}{N}$ of users can fail in an unknown way, then the cooperation gains are limited to what is possible with $N$ trusted users.

\section{PREliminaries}

\section{A. Cooperative Regimes}

The level of cooperation is determined by the bandwidth of the control channel and the quality of the detector. Using these two metrics we can define three regimes of interest:

1) Low bandwidth control channel, Energy detector radios: In this regime, we expect a low bandwidth control channel which is especially true of initial setup stages. Under such a scenario, it is realistic to assume that the radios exchange decisions or summary statistics rather than long vectors of raw data. Furthermore, we assume radios that have no a priori information about the the correlation structure of the signal and hence must integrate the received energy. In [9], it has been shown that with noise uncertainty, energy detectors suffer from a lower bound on the SNR (called $S N R_{\text {wall }}$ ) below which detection is not reliable.

2) Low bandwidth control channel, Detectors utilizing signal statistics: An example of such detectors are cyclo-stationary detectors which utilize the correlation in the signal and hence perform better than energy detectors [10]. However, given the presence of a low bandwidth control channel, only summary statistics can be exchanged.

3) High Bandwidth Control channel, All possible detectors: In this regime, Cognitive Radios can exchange entire raw data and hence sophisticated detection can be performed. In this scenario, we can show that cooperation can enable tightly synchronized radios to collectively overcome the $S N R_{\text {wall }}$.

In this paper we are interested in the first regime since it gives us the lower bound on cooperative performance. 


\section{B. Radio Sensitivity as a metric for Cooperative Gain}

Cognitive Radios must be constrained not to exceed the target probability of harmful interference at the Primary receiver $\left(P_{H I}\right)$. If there are $K$ non-cooperating systems potentially contributing to the interference, then each individual system or network must ensure that its probability of detection is at least $P_{D \text {,system }} \approx 1-\frac{P_{H I}}{K}$ (this can be derived by applying the union bound to the event that any system interferes with the Primary receiver). As the number of cooperating radios $(N)$ in a given Cognitive network is increased, the required probability of detection of an individual radio $P_{D \text {,radio }}$ is reduced as (assuming independent observations at each radio) [11], [12]:

$$
\begin{aligned}
P_{D, \text { radio }} & =1-\sqrt[N]{1-P_{D, \text { system }}} \\
& =1-\sqrt[N]{\frac{P_{H I}}{K}}
\end{aligned}
$$

Viewing this equation on the log scale reveals that $N$ scales as the logarithm of $K$ which implies that an order of magnitude increase in the deployment of non-cooperating systems can be compensated by a linear increase in the number of cooperating users within each system. As $N$ increases beyond $\log K-\log P_{H I}$, the required $P_{D \text {,radio }}$ rapidly approaches 0 .

The reliability of an energy detector depends on the receiver's noise characteristics, the received signal strength, and the length of time that is used for integration. The received signal strength is our focus for two reasons:

- In the presence of noise uncertainty, users below the $S N R_{\text {wall }}$ cannot improve their performance even with infinite integration times.

- Limits on the number of samples available may be imposed by the dwell times of the Primary Users.

Based on this discussion, our model of the radio is simple: given a threshold for the received signal strength $t$, the radio declares that the Primary user is present if and only if the received signal strength is greater than $t$. To meet the target $P_{D, \text { radio }}$, it is necessary that the received signal strength exceed $t$ even in the worst $P_{D \text {, radio }}$ fraction of the fades. Since cooperation makes $P_{D \text {,radio }}$ close to zero, the system as a whole becomes robust to the details of the fading environment.

\section{The Radio Channel}

The Radio channel has three different elements which are important for our analysis:

Distance dependent Path Loss: Path loss forms the most significant portion of the energy loss. A realistic model of cooperative Cognitive usage is a group of users localized in a small area $\left(\sim 1 \mathrm{~km}^{2}\right)$. In such a situation, differences due to path loss are negligible $(.1-.5 \mathrm{~dB})^{4}$. In this paper, we consider a group of Cognitive Radios situated at a distance of $60 \mathrm{~km}$ from a TV transmitter of $100 \mathrm{~kW}$ power. The distance of $60 \mathrm{~km}$ is well beyond the grade B service contour of TV reception [6].

\footnotetext{
${ }^{4}$ Geographically dispersed users would further aid cooperation since some users might be significantly closer to the primary transmitter.
}

Multipath We assume that small scale fading is flat and exhibits a Rayleigh distribution. For Primary user detection, flat fading yields the worst case performance since frequency selectivity provides multiple 'looks' at the same signal. Similarly, Rayleigh fading is considered, since the case of interest is when we cannot count on line of sight between the Cognitive Radio and the Primary transmitter. It is important to note that multipath cannot be relied upon to yield gains (our aim is only to avoid severe multipath losses) since we could easily end up in a deployed scenario where there is Ricean fading.

Shadowing Shadowing on the log scale has been assumed to be normally distributed [13] based on the application of Central Limit Theorem to a large number of small absorptive losses. The standard mechanism to derive the shadowing environment is to take measurements at various locations for a fixed transmitter-receiver separation and attribute the variance in the measurements to shadowing. A naive interpretation would indicate that shadowing can lead to a gain - however it must be realized that the mean received power level in this case has no physical significance. To relate the shadowing to the distance dependent path loss, we used a different model of shadowing where shadowing is viewed as losses via a series of obstacles. For each obstacle, there is a small probability that the obstacle will be missed. Using this model, shadowing is viewed as extra loss beyond the distance dependent path loss. Hence,

$$
Y_{i}= \begin{cases}0 & w \cdot p \cdot 0.2 \\ x d B & w \cdot p \cdot 0.8\end{cases}
$$

and the net shadowing is expressed as:

$$
S=\frac{1}{\sqrt{M}} \sum_{i=1}^{M} Y_{i}
$$

The loss per obstacle was adjusted to fit the variance of the measured log-normal standard variable of around 3.5dB [14]. The resulting value of $M$ (number of obstacles encountered) was 15 while $x$ was $10.25 \mathrm{~dB}$. The Complementary Cumulative Distribution Function (CCDF) of the resulting shadowing random variable is shown in Figure 3 along with the CCDF of multipath.

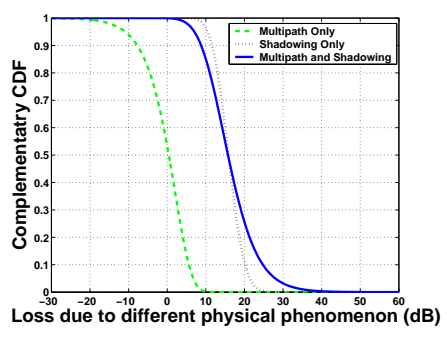

Fig. 3. Complementary CDF of Loss (in $\mathrm{dB}$ ) due to different physical effects.

It is also important to keep in mind that shadowing is notoriously hard to model accurately and its statistics can vary widely with deployment environment. 


\section{GAINS FROM COOPERATION}

\section{A. Impact of number of users}

Using the loss model discussed in the previous section, we simulated the allowed reduction in sensitivity of individual radios as the number of users is increased. This simulation assumes path loss predictions by the NTIA model at a confidence level of $15 \%$ as the nominal distance dependent path loss [15]. This model accounts for losses due to frequency, distance, antenna heights, polarization, surface refractivity, electrical ground constants and climate and hence yields realistic loss levels. Figure 4 shows the change in threshold with increasing number of users under three different effects: multipath only, shadowing only and multipath together with shadowing. We consider gains beyond the nominal path loss as artificial and these should be ignored. When multipath is considered together with shadowing, the threshold asymptotically approaches the nominal path loss. With shadowing alone, the approach is slower due the absence of multipath gains. Half the gain is achieved by using $\sim 10-20$ users, beyond which the gains exhibit a 'law of diminishing returns' as the number of users is increased.

It is important to realize that a single user acting alone must be robust to extremely rare events. These events are not well modelled by the Central Limit theorem, and may in fact not be properly modelled by any single statistical model given the uncertainty that surrounds actual deployment scenarios. At these levels, sensitivity predictions are no better than pure guesswork in the single user context. One of the major advantages of cooperative sensing is that it allows us to have collective robustness to fading while not requiring us to have great faith in the fading model for even moderately uncommon fades. Since $P_{D, \text { radio }}$ is small, only the common fades need to be modelled accurately to get solid quantitative results.

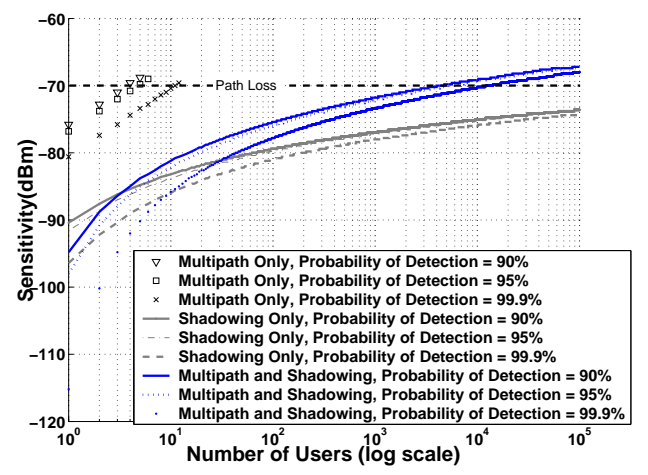

Fig. 4. Sensitivity variation with number of users (Frequency $=800 \mathrm{MHz}$, Distance $=60 \mathrm{~km}, \mathrm{TV}$ transmitter height $=200 \mathrm{~m}, \mathrm{CR}$ height $=3 \mathrm{~m})$. With multipath only, results show an unbounded improvement in threshold as the number of users is increased. Multipath together with shadowing causes the threshold to asymptotically approach the nominal path loss. Shadowing alone gets there slower.

\section{B. Soft versus Hard cooperation}

It has been argued that soft decision combining of sensing results yields gains that are much better than hard decision combining [16]. This is true when radios are tightly synchronized in which case they can collectively overcome the $S N R_{\text {wall }}$. From [9] we know that the physical noise uncertainty gives a lower bound on signal strength that a user can reliably detect. This lower bound is increased further to keep the probability of false alarm tolerable. To understand this better, consider the problem of detecting a signal in additive white Gaussian noise (AWGN) with an noise uncertainty $\alpha$ [9]. For user $i$, our goal is to distinguish between the hypotheses:

$$
\begin{array}{ccc}
\mathcal{H}_{0}: Y_{i}[n]= & \alpha W_{i}[n] & n=1, \ldots, M \\
\mathcal{H}_{s}: Y_{i}[n]= & X[n]+\alpha W_{i}[n] & n=1, \ldots, M
\end{array}
$$

Given that we are using a simple energy detector, the test statistics available under $\mathcal{H}_{0}$ is [17]:

$$
\mathcal{T}\left(Y_{i}\right)=\frac{1}{M} \sum_{j=1}^{M} W_{i}[n]^{2}
$$

Its can be shown that:

$$
\frac{M \mathcal{T}\left(Y_{i}\right)}{\alpha^{2} \sigma_{w}^{2}} \sim \chi_{M}^{2}
$$

For large $M$, this behaves as $\mathcal{N}(M, 2 M)$. Hence we can approximate $\mathcal{T}\left(Y_{i}\right)$ as $\mathcal{N}\left(\alpha^{2} \sigma_{w}^{2}, \frac{\alpha^{4} \sigma_{w}^{4}}{M}\right)$.

If we wish to have a net probability of false alarm $\left(P_{F A}\right)$ to be around 0.14 percent, the threshold should be set 3 standard deviations away from the mean. This places the threshold ${ }^{5}$ at: $\alpha_{\max }^{2} \sigma_{w}^{2}\left(1+\frac{\sqrt{2(9+\ln N)}}{\sqrt{M}}\right)$. The factor $\alpha_{\max }^{2} \sigma_{w}^{2}$ is the worst case noise power.

For soft decoding, we can bound performance by assuming that all the samples are provided to the user with the best channel. In that case the probability of false alarm threshold can be set at: $\alpha_{\max }^{2} \sigma_{w}^{2}\left(1+\frac{3 \sqrt{2}}{\sqrt{M N}}\right)$, where $N$ is the number of users.

To observe the differences between soft and hard decoding we simulated a group of user at a distance of $60 \mathrm{~km}$ from the TV transmitter. The number of users in this group was varied and the effect on radio sensitivity for a $95 \%$ probability of detection was observed. The results of this simulation can be seen in Figure 5. The small difference between hard and soft decision arises from the larger number of samples available in the soft case, but is less than a fraction of a dB.

\section{Shadowing Correlation}

In [11] the received signal is treated as a complex Gaussian process and the effect of correlation is identified. The optimal detector is derived assuming that a single entity has access to all the data and performs optimal detection. These results

\footnotetext{
${ }^{5}$ We need to introduce the tiny $\sqrt{\log N}$ correction term to translate systemlevel false alarm probabilities to radio-level false alarm probabilities.
} 


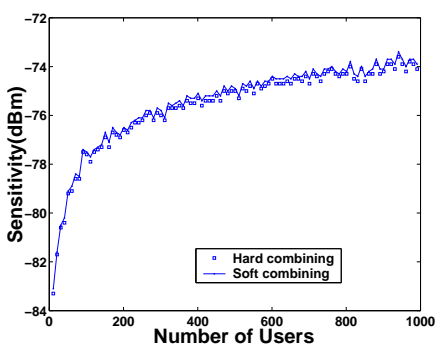

Fig. 5. Radio sensitivity for soft versus hard decoding. The difference between soft and hard decoding is due to the effect of finite number of samples.

show that higher correlation yields a higher probability of false alarm for the same probability of detection. To get a better handle on the correlation problem we make the following observations:

- Multipath at different radios is essentially uncorrelated. Multipath exhibits correlation (both positive and negative) on the scale of $\frac{\lambda}{2}$. Radio placements on this scale can be safely assumed to be uniformly distributed and independent of each other. Hence the net multipath at each radio is uncorrelated.

- Shadowing can display high correlation if two radios are blocked by the same obstacle.

- Constant shadowing correlation is unrealistic. Shadowing correlation displays distance dependence (generally exponentially decreasing with distance) which has been studied extensively [18]. In fact, in certain scenarios, shadowing can be negatively correlated once the distance between radios is increased beyond a certain value [18].

To study the effect of shadowing correlation, we simulated a group of Cognitive users in a line at a distance of $60 \mathrm{~km}$ from a TV transmitter as shown in Figure 6 . The polling entity which is located at the center of this group examines the detection results of users. ${ }^{6}$ The effect of increasing cooperation on the sensitivity threshold of an individual radio can be seen in Figure 7. As a comparison, we also plotted results for constant correlation. It must be noted that we ignore multipath effects in this simulation to prevent favorable multipath gains from swamping the shadowing effects.

All forms of correlation (constant or distance dependent) only serve to increase the number of users required to achieve a given sensitivity reduction. It must be noted that increasing cooperation to compensate for correlated shadowing has limits and cooperative gains in a correlated fading environment are asymptotically lower than in an independent environment. Increased correlation decreases our chances of getting a user with a very good channel and hence more users need to be polled for independent looks at the same random variable. For distance dependent correlation, this translates into a desire to

\footnotetext{
${ }^{6} \mathrm{We}$ choose a linear increase model since the shadowing correlation model as proposed in [18] only predicts one dimensional correlation. Furthermore, we have used the conservative suburban model fit from [18] where the correlation is always positive.
}

poll users which are further away. This effect can be seen in Figure 8. Here, we are interested in studying the sensitivity gains as the number of users and their distance spread is varied. Each set of points represents increased user density. Increasing the number of users for a given distance spread asymptotically reaches a limit which is dependent on the distance spread. A similar effect can be seen in [16] where the probability of missed opportunity does not go to zero in a spatially bounded correlated fading environment.

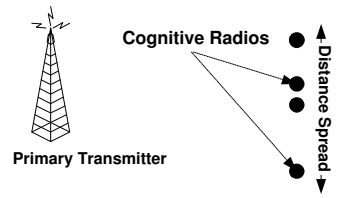

Fig. 6. Simulation setup for distance dependent shadowing correlation.

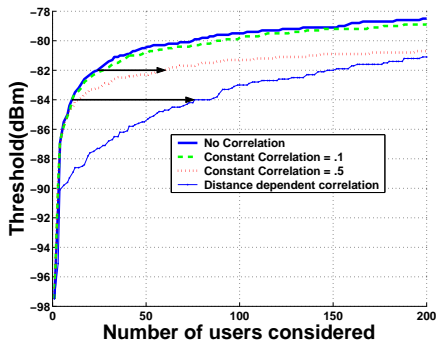

Fig. 7. Sensitivity variation with number of users under different correlation characteristics (no multipath). Correlation causes the system to poll more users to achieve a given threshold.

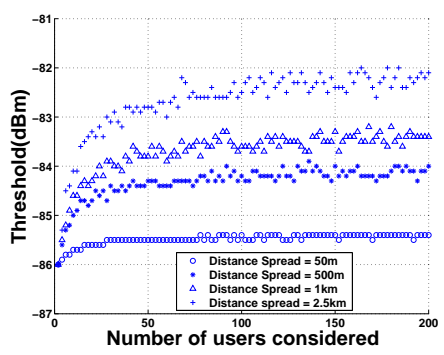

Fig. 8. Comparing the impact of varying users versus varying the distance spread. The plot emphasizes the need for independent samples; gains from increasing the number of users is asymptotically limited in a correlated environment.

\section{IMPACT OF UNTRUSTED USERS}

The results presented so far have established that collectively sensing spectrum availability can deliver tremendous gains even with a small to moderate number of perfectly trusted users, as long as these are far enough apart from each other. These gains are significant enough to justify revisiting the current per-device model of licensing that is used globally. The results so far strongly suggest that devices should be 
regulated on the basis of their provable collective behavior. At this point, a key question emerges - what is the impact of a few malfunctioning devices on the collective? Alternatively, the designer needs to be able to balance the potential gains from admitting another user into the decision-making network against the costs of having an untrusted colleague.

For cooperative sensing, trust issues arise naturally given the usage model:

- Sensing a frequency band, consumes energy and time which may alternatively be diverted to data transmissions. Hence users have a incentive to either not sense at all or to sense for a shorter duration then stipulated.

- For an individual user, there may be a valid reason to report detection results in a certain way. They can either always assert the presence of a Primary user, in which case they deny others the opportunity to take advantage of the available bandwidth, or always deny the presence of Primary users (when users want to use the channel at any cost). We term the two classes of users as always Yes and always No users. These classes of users behave in a predetermined fashion (ie. they fail in predictable ways).

- Radios may fail in unpredictable ways or be simply malicious. For such users, we need to budget for worse case performance.

\section{A. Impact of Always No and Always Yes Adversaries}

Dealing with Always No liars does not require knowledge of the number of liars in the system. Always No users always report the absence of a Primary user and hence they effectively reduce the number of actual users in the system. This effect is captured in Figure 9. Twice as many users are needed to achieve the same threshold with 50\% Always No liars than with a fully trusted system.

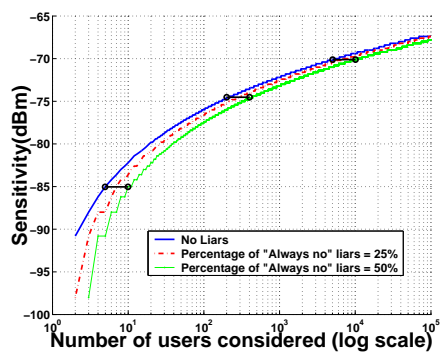

Fig. 9. Sensitivity Variation with Always No adversaries. Twice as many users are needed to achieve the same threshold with $50 \%$ Always No liars than with a correctly operating system.

Similarly, if we know that there are exactly y Always Yes users, then we can signal presence of a Primary user only when $y+1$ users detect a Primary. Once again, it is like having fewer users. However, the situation changes drastically when we only have a bound on the proportion of Always Yes users as the next section will show.

\section{B. Dealing with malicious Adversaries}

Malicious adversaries are impossible to predict reliably. For such adversaries we need to budget for worst case perfor- mance. Assume that we have $N$ users of which a fraction $(\alpha \in[0,1])$ may be Always Yes liars. To deal with these liars, we can set the detection threshold at $\beta N$ where $\beta>\alpha$ ie. we declare that a Primary user is present if $\beta N$ Cognitive Radios see the Primary user.

The problem arises when these Always Yes liars actually behaving as Always No liars in a system configured to be robust to Always Yes liars. Not only do they effectively reduce the number of real users in the system to $N(1-\alpha)$, but they also now require a fraction $\frac{\beta}{1-\alpha}$ of the trustworthy users to detect the signal.

Explicitly, the resulting probability of detection for a given threshold $t$ is given by:

$$
P_{d, t}=1-\sum_{i=0}^{\beta N-1}\left(\begin{array}{c}
N(1-\alpha) \\
i
\end{array}\right) F(t)^{i}(1-F(t))^{N(1-\alpha)-i}
$$

where $F(t)$ is the CDF of the received signal strength.

For a $95 \%$ overall detection probability, the corresponding threshold values can be seen in Figure 10. The individual sensitivity threshold tolerable for a group with a fraction of $\frac{1}{N}$ liars is the same as that achievable by a trusted system with $N$ users. This threshold forms an upper bound even when the actual number of users is increased beyond $N$. Hence for $1 \%$ liars, the final achievable threshold is $-76 \mathrm{dBm}$ which is the threshold for a trusted system with 100 users. The mathematical intuition for this result can be obtained by seeing that even when the number of users is large, for a fraction $\frac{\beta}{1-\alpha}$ of them to detect the primary requires that the threshold be such that $F(t) \leq 1-\frac{\beta}{1-\alpha}$ and so the threshold $t$ must be small enough and can not be made any larger than that. When the proportion of malicious users is high, it does require a moderately large number of users to reach these asymptotic limits.

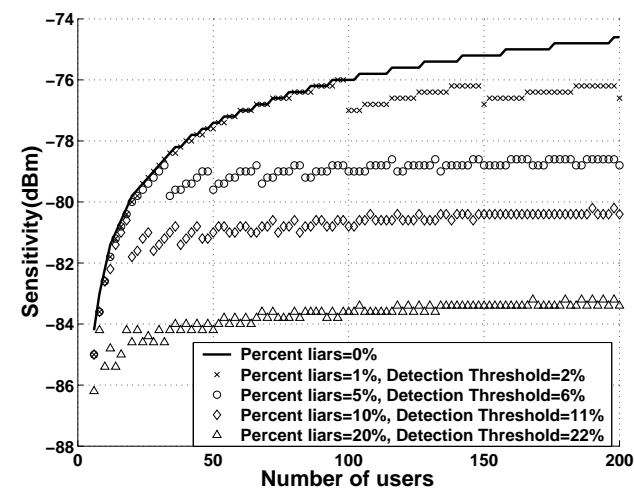

Fig. 10. Sensitivity Variation with malicious Always Yes adversaries. The threshold achievable for a group with a fraction of $\frac{1}{N}$ liars is the same as that achievable by a trusted system with $N$ users

\section{Conclusions}

In this paper, we have suggested light-weight cooperation as a means to reduce the sensitivity requirements on an individual 
Cognitive Radio. Exploiting cooperation among multiple users may be the only mechanism to achieve a target system-level probability of detection in the case when each Cognitive radio faces an $S N R_{\text {wall }}$ below which it is unable to reliably detect a Primary. With enough trusted cooperation, we only need to be sensitive enough to deal with the nominal path loss. However, this requires cooperation among users facing more or less independent fading. Shadowing is likely to be correlated across space. This correlation can be dealt with by increasing the number of users up to certain sensitivity levels. When correlation is distance-dependent, cooperation is desired among more distant users. Increasing the number of users in a distance-dependent correlated setting is asymptotically limited by the distance spread. Furthermore, a hard decision scheme performs as well as a soft decision, with small differences arising from finite number of samples.

Even so, trust is critical for such a cooperative systems to operate reliably. Users that fail in a known fashion (assert/deny the presence of a Primary user), can be compensated for, by increasing the number of users polled. Unfortunately, malicious users or users that fail in unknown ways impose an upper bound on achievable sensitivity reductions. As a rule of thumb, if one out of every $N$ users is untrustworthy, then the sensitivity of an individual receiver may not be reduced below what is possible with $N$ trusted users.

\section{REFERENCES}

[1] Robert W. Broderson, Adam Wolisz, Danijela Cabric, Shridhar Mubaraq Mishra, and Daniel Willkomm. White paper: CORVUS: A Cognitive Radio Approach for Usage of Virtual Unlicensed Spectrum. Technical report, 2004.

[2] Spectrum policy task force report. Technical Report 02-135, Federal Communications Commision, Nov 2002.

[3] Dupont Circle Spectrum Utilization During Peak Hours. Technical report, The New America Foundation and The Shared Spectrum Company, 2003.

[4] Unlicensed Operation in the TV Broadcast Bands and Additional Spectrum for Unlicensed Devices Below $900 \mathrm{MHz}$ in the $3 \mathrm{GHz}$ band. NOTICE OF PROPOSED RULEMAKING 04-186, Federal Communications Commision, May 2004

[5] N. Hoven and A. Sahai. Power scaling for cognitive radio. In Proc. of the WirelessCom 05 Symposium on Signal Processing, 2005.

[6] Longley-Rice Methodology for evaluating TV Coverage and Interference. OET Bulletin 69, Office of Engineering and Technology (OET), Federal Communications Commision, Feb 2004.

[7] D. Tse and P. Viswanath. Fundamentals of Wireless Communications. Cambridge University Press, 2005.

[8] A. Sendonaris, E. Erkip, and B. Aazhang. User Cooperation DiversityPart i: System Description. IEEE Transactions on Communications, 51(11), November 2003.

[9] R. Tandra and A. Sahai. Fundamental limits on detection in low SNR under noise uncertainty. In Proc. of the WirelessCom 05 Symposium on Signal Processing, 2005.

[10] D. Cabric, S. M. Mishra, and R. W. Brodersen. Implementation issues in spectrum sensing for cognitive radios. In Asilomar Conference on Signals, Systems, and Computers, 2004.

[11] T. Weiss, J. Hillenbrand, and F. Jondral. A diversity approach forthe detection of idle spectral resources in spectrum pooling systems. In Proc. of the 48th Int. Scientific Colloquium, Ilmenau, Germany, 2003.

[12] A. Ghasemi and E. S. Sousa. Collaborative Spectrum Sensing for Opportunistic Access in Fading Environments. To appear in Proc. of the 1st IEEE Conference on Dynamic Spectrum Management (DySPAN05), 2005.

[13] T. S. Rappaport. Wireless Communications: Principles and Practice. Prentice Hall, 2002.
[14] Chris Weck. Validate Field Trials of Digital Terrestrial Television (dvbt). Technical report, Institut fr Rundfunktechnik GmbH Rundfunksystementwicklung Mnchen, Germany.

[15] Irregular Terrain Model (ITM) (Longley-Rice). Technical report, U.S. Department of Commerce NTIA.

[16] E. Vistotsky, S. Kuffner, and R. Peterson. On Collaborative Detection of TV Transmissions in Support of Dynamic Spectrum Sharing. To appear in Proc. of the 1st IEEE Conference on Dynamic Spectrum Management (DySPAN05), 2005.

[17] A. Sahai, N. Hoven, and R. Tandra. Some Fundamental Limits on Cognitive Radio. In Allerton Conference on Communication, Control, and Computing, 2003.

[18] M. Gudmundson. Correlation model for shadow fading in mobile radio systems. Electronic Letters, 27(23):2145-2146, 1991. 